\title{
Trajetória científica da teoria dos escalões superiores: estudo das publicações
}

A Teoria dos Escalões Superiores (TES) preconiza que os resultados e processos estratégicos organizacionais são uma função das características gerenciais dos gestores superiores ou gestores de topo. Desde a publicação da TES, pesquisadores ampliaram estudos sobre como as características observáveis da Equipe de Gestão de Topo (EGT) podem ser usadas para explicar a estratégia corporativa. Esta pesquisa tem por finalidade traçar a trajetória das publicações científicas da Teoria dos Escalões Superiores (TES), de modo a evidenciar a epistemologia e as publicações posteriores. A seleção dos artigos segue uma sequência de cinco etapas, quais sejam: definição de palavras-chave; seleção das bases de dados; busca nas bases; leitura dos títulos; e leitura dos resumos. Notou-se que a Teoria é constituída com origem na Teoria Comportamental, no aspecto da escolha estratégica e foco primário na coalizão dominante ao invés de estritamente no executivochefe. Destacam-se dez características usuais entre os trabalhos: educação e heterogeneidade/homogeneidade da equipe.

Palavras-chave: Teoria dos Escalões Superiores; Trajetória epistemológica; Publicações científicas.

\section{Scientific trajectory of the upper echelons theory: study of publications}

\begin{abstract}
The Upper Echelons Theory (UET) states that organizational outcomes and strategic processes are a function of the managerial characteristics of top managers or top managers. Since the publication of TES, researchers have expanded studies on how observable characteristics of the Top Management Team (TMT) can be used to explain corporate strategy. This research aims to trace the trajectory of the scientific publications of the Upper Echelons Theory (UET), in order to highlight epistemology and later publications. The selection of the articles follows a sequence of five steps, namely: definition of keywords; selection of databases; search the bases; reading titles; and reading summaries. It was noted that the Theory is constituted from Behavioral Theory, in the aspect of strategic choice and primary focus in the dominant coalition rather than strictly in the chief executive. Ten characteristics typical of the work are highlighted: education and heterogeneity/homogeneity of the team.
\end{abstract}

Keywords: Upper Echelons Theory; Epistemological trajectory; Scientific publications.

Topic: Teorias Contábeis

Reviewed anonymously in the process of blind peer.
Received: $15 / 10 / 2019$

Approved: 21/12/2019
Paula de Souza Michelon (iD)

Universidade Federal de Santa Catarina, Brasil http://lattes.cnpq.br/4324204678711922

http://orcid.org/0000-0002-8174-8859

pauladesouza1604@gmail.com

Rogério João Lunkes (iD

Universidade Federal de Santa Catarina, Brasil http://lattes.cnpq.br/6241003358183170

http://orcid.org/0000-0003-4232-5746

rogeriolunkes@hotmail.com

Antonio Cezar Bornia (iD

Universidade Federal de Santa Catarina, Brasil

http://lattes.cnpq.br/1042018203108549

http://orcid.org/0000-0003-3468-7536

cezar.bornia@gmail.com 


\section{INTRODUÇÃO}

A tomada de decisão é um elo importante na administração das empresas. A gestão das empresas é construída com as decisões de diferentes níveis, sejam eles de grande importância ou não. A rapidez, a precisão e a execução da tomada de decisão afetam diretamente a qualidade da decisão que, de certa forma, tem grande influência sobre o efeito da gestão das empresas e sobre a eficiência da equipe (GUO et al., 2011).

A Teoria dos Escalões Superiores (TES), difundida por Hambrick et al. (1984), sugere que os resultados e processos estratégicos organizacionais são uma função das características gerenciais dos gestores superiores ou gestores de topo. A principal noção da Teoria é que as escolhas estratégicas, ao contrário das decisões operacionais, são mais oriundas de fatores comportamentais do que de cálculo mecânico para a otimização econômica. Como decorrência, as escolhas estratégicas geralmente possuem uma grande quantidade de componentes comportamentais e, em alguma medida, refletem as idiossincrasias dos tomadores de decisão (CHUANG et al., 2007; CHUANG et al., 2009).

Desde a publicação do trabalho seminal de Hambrick et al. (1984), pesquisadores ampliaram estudos sobre como as características observáveis da Equipe de Gestão de Topo (EGT) podem ser usadas para explicar a estratégia corporativa. Um pressuposto chave destas pesquisas é que as características observáveis são indicadores demográficos úteis de experiências individuais, habilidades, valores, estilos cognitivos e fontes de informação (JEHN et al., 1999). Grande parte dessa pesquisa explorou como a diversidade demográfica da EGT ou a heterogeneidade, termos utilizados de forma intercambiável, influenciam a inovação estratégica (FINKELSTEIN et al., 1996).

A sugestão de Hambrick et al. (1984) de que as características demográficas servem como proxies para as crenças, valores e cognições dos gestores abriu o caminho para um grande número de estudos empíricos subsequentes. Embora não sejam substitutos perfeitos das construções subjacentes, as variáveis demográficas oferecem a vantagem de serem objetivas, testáveis e abrangentes (HAMBRICK et al., 1984). Estudos anteriores relacionaram os dados demográficos da alta administração à estratégia (MICHEL et al., 1992; THOMAS et al., 1991), mudança estratégica ou persistência (FINKELSTEIN et al., 1990; WIERSEMA et al., 1992) e postura de diversificação (MICHEL et al., 1992)

Apesar das vantagens da objetividade e da disponibilidade de dados sobre as informações demográficas da EGT, a perspectiva da TES tem sido criticada por ignorar a 'caixa preta', na qual as variáveis de mediação assumem a função de vínculo entre dados demográficos e resultados (PETTIGREW, 1992). Lawrence (1997) descreveu que o problema é a utilização de variáveis demográficas para representar conceitos subjetivos (atitudes, cognições e valores) sem fornecer uma justificativa para o porquê esta é uma abordagem válida.

Por outro lado, pesquisadores têm defendido o uso de dados demográficos considerando: (i) as vantagens de objetividade, compreensão, validade de conteúdo, reprodutibilidade e disponibilidade de dados, pois os altos executivos geralmente não estão dispostos a se submeter a baterias de testes psicológicos (FINKELSTEIN et al., 1996); e (ii) a investigação baseada na TES deu resultados estatisticamente 
significativos no que diz respeito à identificação de relações entre as características demográficas, estratégias e desempenho da EGT. Assim, a acumulação de estudos significativos fornece uma justificativa empírica convincente para o uso continuado da perspectiva dos escalões superiores (GOLL et al., 2005).

De todo modo, a TES proporcionou uma investida para a pesquisa empírica, argumentando que as características demográficas das equipes de alta direção são boas proxies para os traços subjacentes e os processos cognitivos dos principais executivos (WIERSEMA et al., 1992; HAMBRICK et al., 1996; CARPENTER et al., 2001; HAO et al., 2012; DÍAZ-FERNÁNDEZ et al., 2014).

Neste sentido, o presente artigo tem por finalidade traçar a trajetória das publicações científicas da TES, de modo a evidenciar de onde a Teoria veio (epistemologia) e para onde ela está indo (publicações posteriores). Esta pesquisa é relevante, pois permite identificar as contribuições que proporcionaram a estruturação da TES, bem como com a identificação do rumo teórico e empírico.

\section{METODOLOGIA}

A seleção dos artigos para estruturação da trajetória científica segue uma sequência de cinco etapas, quais sejam: i) definição de palavras-chave; ii) seleção das bases de dados; iii) busca dos artigos nas bases de dados selecionadas; iv) leitura dos títulos; e v) leitura dos resumos. Tal sequência metodológica para construção do conjunto de artigos seguiu uma adaptação do Proknow-C (Knowledge Development ProcessConstructivist), um processo estruturado para a construção de conhecimento pelo pesquisador sobre um tópico ou questão (DUTRA et al., 2015; ENSSLIN et al., 2014). A diferença é que nesta pesquisa não houve delimitação de artigos conforme citações no Google Scholar. Assim, todos os títulos dos artigos foram lidos e filtrados, excluindo-se apenas as duplicidades.

Primeiramente, filtraram-se as expressões 'escalão superior' ou 'equipe de gestão de topo', bem como 'upper echelon' ou 'top management team', nos títulos, resumos e palavras-chave dos artigos. A busca dos artigos nas seis bases de dados ocorreu no período de 20 de março de 2017. Não se restringiu o período de publicações, ou seja, os artigos publicados até 20 de março de 2017 poderiam ser selecionados. Nesta pesquisa retornaram 3.118 artigos publicados nas bases de dados EBSCO Academic Search Premier, ISI Web of Science, Science Direct, Scopus - Elsevier e Wiley Online Library e Scientific Periodicals Eletronic Library SPELL, dos quais, 1.355 foram eliminados por serem redundantes, ou seja, publicados em mais de uma das bases de dados.

Foram lidos os títulos dos 2.043 artigos, dos quais foram eliminados 1.564 artigos por não se referirem à Teoria pesquisada, resultando em 479 artigos para leitura do título. Desse total, resultaram 195 com títulos alinhados. Realizou-se a leitura e análise dos resumos dos 195 artigos, com o intuito de verificar a aderência quanto ao tema definido. A leitura permitiu identificar 57 alinhados à Teoria, dos quais 15 são teóricos.

\section{RESULTADOS E DISCUSSÃO}

A trajetória da Teoria dos Escalões Superiores compreende a análise das publicações científicas 
antecedentes e precedentes de sua publicação consolidada em 1984. A trajetória epistemológica compreende a análise das publicações antecedentes, que contribuíram para a construção da Teoria. A trajetória precedente envolve as publicações posteriores à Teoria que buscaram aplica-la e revisa-la.

\section{Trajetória epistemológica da Teoria dos Escalões Superiores}

A primeira etapa consiste em delinear a trajetória epistemológica da Teoria dos Escalões Superiores (TES). O objetivo é identificar os principais autores e contribuições que formaram a referida Teoria. Em outras palavras, constitui na organização dos artigos preliminares para compor os antecedentes da TES. Na análise dos artigos percebeu-se a recorrência de um artigo considerado propulsor da Teoria: o artigo de Hambrick et al. (1984), com 9.400 citações, sob o título "Upper echelons: the organization as a reflection of its top managers".

Quadro 1: Trajetória Epistemológica da Teoria dos Escalões Superiores.

\begin{tabular}{|c|c|c|}
\hline Ano & Autor(es) & Principal contribuição \\
\hline 1955 & Simon & \multirow{2}{*}{$\begin{array}{l}\text { Racionalidade limitada - os gestores são confrontados } \\
\text { com muito mais informações do que eles podem lidar. }\end{array}$} \\
\hline \multirow[b]{2}{*}{1958} & March e Simon & \\
\hline & Dearborn e Simon & $\begin{array}{l}\text { Antecedentes funcionais - estão relacionados à } \\
\text { interpretação de problemas críticos em negócios } \\
\text { complexos. }\end{array}$ \\
\hline 1959 & Penrose & $\begin{array}{l}\text { Visão Baseada em Recursos - argumentou que o sucesso } \\
\text { da empresa ocorre, sobretudo, em função dos seus } \\
\text { recursos competitivos internos. }\end{array}$ \\
\hline 1962 & Chandler & $\begin{array}{l}\text { Perspectiva da escolha estratégica - as escolhas gerenciais } \\
\text { desempenham um papel importante nas ações e } \\
\text { resultados organizacionais. }\end{array}$ \\
\hline 1963 & Cyert e March & $\begin{array}{l}\text { Coalizão dominante - as características centrais das } \\
\text { equipes de alta gerência melhora a confiança na previsão } \\
\text { sobre as estratégias empresariais. }\end{array}$ \\
\hline 1971 & Andrews & \multirow{2}{*}{$\begin{array}{l}\text { Perspectiva da escolha estratégica - alta administração } \\
\text { deve alinhar as oportunidades e ameaças no ambiente } \\
\text { externo com os pontos fortes e fracos de uma organização. }\end{array}$} \\
\hline 1972 & Child & \\
\hline 1982 & Song & $\begin{array}{l}\text { Variáveis demográficas - existia relação entre as } \\
\text { características da equipe de escalões superiores e as } \\
\text { estratégias de diversificação de empresas. }\end{array}$ \\
\hline 1983 & Pfeffer & $\begin{array}{l}\text { Variáveis demográficas - o desempenho geral da empresa } \\
\text { e os outros resultados são afetados por características } \\
\text { demográficas. }\end{array}$ \\
\hline \multirow{2}{*}{1984} & Wernerfelt & $\begin{array}{l}\text { Visão Baseada em Recursos - equipe de direção como um } \\
\text { recurso estratégico da empresa. }\end{array}$ \\
\hline & Hambrick e Mason & $\begin{array}{l}\text { Perspectiva dos Escalões Superiores - a organização como } \\
\text { reflexo de seus principais executivos. }\end{array}$ \\
\hline
\end{tabular}

Assim, a partir de Hambrick et al. (1984), foram selecionados os trabalhos anteriores que, de certo modo, participaram da construção de algum aspecto da Teoria. Foram encontrados 12 artigos publicados. $\mathrm{O}$ Quadro 1 resume as principais contribuições no desenvolvimento da Perspectiva dos Escalões Superiores, em ordem cronológica por autor(es), até culminar na Teoria, difundida na década de 1980, por Hambrick et 
al. (1984).

Simon (1955), March et al. (1958) e Cyert et al. (1963) contribuíram com os conceitos da Teoria Comportamental. Simon (1955) propôs racionalidade limitada para se referir ao fato de que os gestores fazem escolhas satisfatórias ao invés de buscarem estratégias de otimização, pois lidam com informações limitadas, tempo e habilidades intelectuais (LI, 2016). Para os estudiosos da Teoria Comportamental, racionalidade limitada, metas múltiplas e conflitantes e níveis de aspiração variados servem para restringir na medida em que decisões complexas podem ser tomadas numa base tecno-econômica. Em síntese, quanto mais complexa for a decisão, mais a Teoria Comportamental é aplicável à TES (HAMBRICK et al., 1984).

$\mathrm{Na}$ TES, a racionalidade limitada está presente na premissa de que as limitações humanas influenciam a percepção, a avaliação e as decisões sobre os problemas organizacionais e, por desfecho, impactam as escolhas e o comportamento da organização. Por conseguinte, esta premissa motivou pesquisadores nos campos da psicologia social e organizacional, desenvolvendo um grande debate (DÍAZ-FERNÁNDEZ et al., 2014).

Por meio dessa relação entre as limitações humanas, escolhas e o comportamento organizacional, alguns estudiosos pesquisaram a respeito das associações entre características observáveis - também chamada demográficas - dos gestores e os resultados organizacionais. Dearborn et al. (1958) descreveram que os backgrounds funcionais dos gestores estavam relacionados à sua interpretação de problemas críticos em negócios complexos. Décadas depois, o trabalho de Walsh (1988) apontou que as estruturas de crenças gerenciais podem ser mais complexas do que posto por Dearborn et al. (1958).

A perspectiva da escolha estratégica foi proposta por Chandler (1962), Andrews (1971) e Child (1972), com a abordagem de que as decisões e as escolhas da alta gerência afetam o desempenho da empresa. Enquanto a teoria da ecologia populacional sugeria que as organizações são inerciais e que o ambiente desempenha um papel chave na seleção das organizações que sobrevivem, ou seja, os gestores tenham pouca influência nos resultados organizacionais (HANNAN et al., 1977). No outro extremo estava a perspectiva de escolha estratégica, a qual sugere que as escolhas gerenciais desempenham um papel importante nas ações e resultados organizacionais (CHANDLER, 1962; ANDREWS, 1971). De acordo com essa perspectiva, a tarefa mais importante da alta administração é alinhar as oportunidades e ameaças no ambiente externo com os pontos fortes e fracos de uma organização (ANDREWS, 1971; CHILD, 1972).

Um conceito importante da Teoria dos Escalões Superiores é o foco primário na equipe de alta gerência ao invés de estritamente no executivo-chefe. Isso quer dizer que, na maioria das vezes, a administração é um esforço compartilhado em que uma coalizão dominante de executivos (CYERT et al., 1963) forma coletivamente os resultados organizacionais (FINKELSTEIN et al., 1990).

Song (1982) e Pfeffer (1983) foram os primeiros a reconhecerem que as variáveis demográficas dos gestores tinham potencial como variáveis causais no estudo dos resultados organizacionais (WALLY et al., 2001; CARPENTER et al., 2006). Song (1982) mostrou que existia relação entre as características da equipe de escalões superiores e as estratégias de diversificação de empresas (CARPENTER et al., 2006). Pfeffer (1983) identificou o desempenho geral da empresa e outros resultados como sendo afetados por características 
demográficas.

Penrose (1959) e Wernerfelt (1984) contribuíram com a abordagem que ficou conhecida como Visão Baseada em Recursos (VBR), no sentido de que a equipe de direção figura como um recurso estratégico da empresa. Primeiramente, Penrose (1959) argumentou que o sucesso da empresa ocorre, sobretudo, em função dos seus recursos competitivos internos (WERNERFELT, 1984). A VBR enfatiza que a fonte da vantagem competitiva consiste, primariamente, nos recursos valiosos, escassos, inimitáveis desenvolvidos e controlados pelas empresas e, apenas em segundo plano, na estrutura das indústrias (BARNEY, 1991). Os antecedentes e a experiência da equipe de escalões superiores, tais como a experiência internacional e os relacionamentos de rede, representam alguns desses recursos (CASTANIAS et al., 2001).

Hambrick et al. (1984) estenderam essas diversas ideias na construção da perspectiva de escalões superiores. Em sua opinião, a organização torna-se um reflexo de seus principais executivos, e as características e o funcionamento da equipe de alta gerência têm um potencial muito maior para prever resultados organizacionais do que as características do CEO (HAMBRICK, 2007). O que os autores trouxeram de novo com a Teoria dos Escalões Superiores é a influência das características gerenciais observáveis na tomada de decisão, isto é, variáveis demográficas. Estas são os indicadores dos valores e da base cognitiva que os gestores trazem consigo ao se depararem com uma situação administrativa (PULEO et al., 1998).

O modelo da Teoria tem duas partes interligadas: (i) os executivos agem com base em suas interpretações personalizadas das situações estratégicas que enfrentam, e (ii) estas interpretações personalizadas são uma função de experiências, valores e personalidades dos executivos (HAMBRICK, 1987). Destarte, na medida em que os 'dados' gerenciais influenciam as escolhas estratégicas esses 'dados' também influenciarão as escolhas associadas à implementação da estratégia de uma empresa e, por meio da implementação, ao desempenho organizacional (PULEO et al., 1998).

\section{Trajetória precedente da Teoria dos Escalões Superiores}

A trajetória das publicações referentes à TES se inicia em 1984, após sua consolidação. Dos 57 artigos alinhados à Teoria publicados a partir do referido ano, 15 propuseram quadros teóricos em distintas perspectivas ou revisaram a Teoria com a finalidade de encontrar oportunidades para futuras pesquisas.

Hambrick (1987), propôs um modelo para a avaliação sistemática e remodelação das equipes de gestão de topo. Alderson (1993) apresentou as competências da equipe de gestão identificadas pelos principais executivos como essenciais para o sucesso. Krishnan et al. (1998) construíram um arcabouço teórico na área de liderança de escalões superiores. Clark et al. (2007) forneceram um quadro teórico das dimensões processuais da alta administração. Deng (2008) relacionou as características da Equipe de Gestão de Topo (EGT) com a adaptabilidade estratégica da EGT. Weihui et al. (2008) apresentaram um modelo multiagente para analisar a composição, configuração e efetividade da EGT. Guo et al. (2011) construíram um modelo de decisão estratégica ligando a EGT e o desempenho da empresa. Yu-Hong et al. (2013) desenvolveram um modelo do mecanismo de discrição gerencial da EGT para decisão estratégica. 
Quadro 2: Principais características demográficas analisadas após 1984.

\begin{tabular}{|c|c|c|c|c|c|c|c|c|c|c|}
\hline \multirow[b]{2}{*}{ Autor( } & \multicolumn{10}{|c|}{ Ca racterísticas Demográficas } \\
\hline & 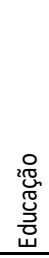 & $\stackrel{.00}{E}$ & 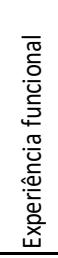 & 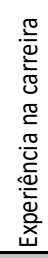 & 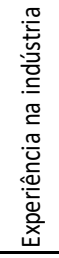 & 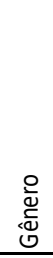 & 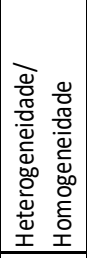 & $\begin{array}{l}\frac{1}{0} \\
\frac{\pi}{0}\end{array}$ & 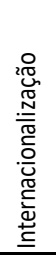 & 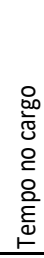 \\
\hline \multicolumn{11}{|c|}{ Chaganti e Sambharya (1987) } \\
\hline \multicolumn{11}{|c|}{ Finkelstein e Hambrick (1990) } \\
\hline \multicolumn{11}{|c|}{ Bantel (1993) } \\
\hline \multicolumn{11}{|c|}{ Hambrick, Cho e Chen (1996) } \\
\hline \multicolumn{11}{|c|}{ Elron (1997) } \\
\hline \multicolumn{11}{|l|}{ Puleo e Marlin (1998) } \\
\hline \multicolumn{11}{|c|}{ Athanassiou e Nigh (1999) } \\
\hline \multicolumn{11}{|c|}{ Pegels e Yang (2000) } \\
\hline \multicolumn{11}{|c|}{ Wallye Becerra (2001) } \\
\hline \multicolumn{11}{|c|}{ Herrmann e Datta (2005) } \\
\hline \multicolumn{11}{|c|}{ Cho e Hambrick (2006) } \\
\hline \multicolumn{11}{|c|}{ Chuang, Nakatani e Zhou (2007) } \\
\hline \multicolumn{11}{|c|}{ Kauer, Waldeck e Schäffer (2007) } \\
\hline \multicolumn{11}{|c|}{ Goll, Johnson e Rasheed (2008) } \\
\hline \multicolumn{11}{|c|}{ Koufopoulos et al. (2008) } \\
\hline \multicolumn{11}{|c|}{ Gu (2008) } \\
\hline \multicolumn{11}{|c|}{ Cannella Júnior, Park e Lee (2008) } \\
\hline \multicolumn{11}{|c|}{ Naranjo-Gil, Hartmann e Maas (2008) } \\
\hline \multicolumn{11}{|c|}{ Gallén (2009) } \\
\hline \multicolumn{11}{|l|}{ Ping (2008) } \\
\hline \multicolumn{11}{|c|}{$\begin{array}{l}\text { Es cribá-Es te ve, Sá nchez-Peina do e } \\
\text { Sá nchez-Peinado (2009) }\end{array}$} \\
\hline $\begin{array}{l}\text { Pa tzelt, Knyphausen } \\
(2009)\end{array}$ & & & & & & & & & & \\
\hline Chuang, Nakatani e & & & & & & & & & & \\
\hline Abebe (2010) & & & & & & & & & & \\
\hline Amason, Liu e Fu (20 & & & & & & & & & & \\
\hline $\begin{array}{l}\text { Camelo, Fernández- } \\
(2010)\end{array}$ & & & & & & & & & & \\
\hline Chen, Hsu e Huang ( & & & & & & & & & & \\
\hline Awa et al. (2011) & & & & & & & & & & \\
\hline Boerner, Linkohre Ki & & & & & & & & & & \\
\hline Kwee, Bosch e Volbe & & & & & & & & & & \\
\hline Ca i, Liu e Yu (2013) & & & & & & & & & & \\
\hline Bany-Ariffin et al. ( 2 & & & & & & & & & & \\
\hline $\begin{array}{l}\text { Díaz-Fernández, Gon } \\
\text { Pawlak (2014) }\end{array}$ & & & & & & & & & & \\
\hline Yuan, Guo e Fang (2C & & & & & & & & & & \\
\hline $\begin{array}{l}\text { Díaz-Fernández, Gon } \\
\text { Simonetti (2015) }\end{array}$ & & & & & & & & & & \\
\hline Li, Zhang e Zhang (2C & & & & & & & & & & \\
\hline Acar (2016) & & & & & & & & & & \\
\hline Li, Tseng e Chen (201 & & & & & & & & & & \\
\hline Li (2016) & & & & & & & & & & \\
\hline Guo, Pang e Li (2017) & & & & & & & & & & \\
\hline
\end{tabular}

Carpenter et al. (2004), Hambrick (2007), Hao et al. (2012), Hiebl (2014), Serra et al. (2014) e Ping et al. (2016) revisaram a TES com o propósito de identificar oportunidades para pesquisas subsequentes. Os demais 42 artigos foram enquadrados como empíricos, isto é, testaram a relação das características dos gestores com outras variáveis. Para traçar a trajetória dessas publicações empíricas atinentes à TES, elaborou-se o Quadro 2 com as principais características demográficas exploradas pelos pesquisadores a partir de 1984.

Primeiramente, observa-se o acréscimo de estudos realizados envolvendo a TES na terceira década, uma vez que na primeira década de análise foram tabulados 4 (1987 a 1996) e na segunda 8 estudos (1997 a 
2006). Ou seja, nota-se o aumento da percepção e do interesse dos pesquisadores em verificar a influência das características dos gestores em diversos aspectos.

A análise dos artigos permitiu a identificação de diversas características. Mas, o Quadro 2 apresenta aquelas que foram utilizadas por no mínimo dois artigos selecionados. Nesse contexto, pode-se perceber que existem dez características usuais entre os trabalhos referentes a TES, com destaque para a educação e a heterogeneidade/homogeneidade da EGT, presentes em mais de $50 \%$ dessas pesquisas.

A educação ou o nível educacional da EGT tem sido estudado por pesquisadores da TES como um importante indicador demográfico que afeta a escolha estratégica e os resultados organizacionais (HAMBRICK et al., 1984). É uma característica demográfica que se refere à maior realização educacional de um indivíduo. No estudo de Camelo et al. (2010) os resultados mostram que um nível educacional mais alto da EGT tem um efeito positivo e direto no desempenho da inovação.

O nível educacional dos gestores foi associado à orientação cognitiva e à base de conhecimento (HERMANN et al., 2005). Entende-se que gestores mais educados possuem conhecimento avançado e maior habilidade cognitiva. Isso, por sua vez, poderia aumentar sua capacidade de tolerar ambiguidade, absorver novas informações e analisar, resolver e implementar soluções para problemas complexos (WALLY et al., 2001; BANY-ARIFFIN et al., 2014).

À medida que a expansão dos negócios da multinacional fora dos Estados Unidos cresce e os vínculos entre suas unidades de negócios internacionais se intensificam, a demanda por expertise de negócios internacionais dentro da equipe aumenta, os membros da EGT compartilham conhecimento mútuo de negócios internacionais e aumenta a densidade da rede (ATHANASSIOU et al., 1999).

Adicionalmente, os resultados de um estudo realizado com gestores dos Estados Unidos indicam que as empresas com níveis mais elevados de diversificação internacional tendem a ter equipes caracterizadas por um nível educacional mais elevado. Ademais, os resultados indicam que as relações entre as características da EGT e a diversificação internacional são mais dominantes em empresas de melhor desempenho do que em empresas de baixo desempenho (HERRMANN et al., 2005).

Os achados da pesquisa feita com gestores de 52 empresas multinacionais dos Estados Unidos na Europa indicam que maior posse organizacional da equipe foi positivamente associada à especialização funcional e negativamente associada com aumentos na diversificação internacional. As perspectivas cognitivas dos membros de uma equipe de alta gerência refletidas nas características demográficas da equipe influenciam o grau de mudança na diversificação internacional da amostra (WALLY et al., 2001).

No estudo de Naranjo-Gil et al. (2008) feito com 92 EGT completas de hospitais da Espanha, os resultados mostraram que a heterogeneidade da EGT relacionada ao trabalho modera a relação entre mudança estratégica e desempenho operacional. Nenhum efeito moderador é encontrado para a heterogeneidade não relacionada ao trabalho da EGT. Adicionalmente, Li et al. (2015), encontraram que há uma relação não-linear entre a heterogeneidade de EGT e o desempenho corporativo que depende do grau de diversificação das empresas de capital aberto da China.

O efeito da heterogeneidade sobre o desempenho sobre o desempenho de companhias aéreas, em 
termos de mudanças na participação de mercado e nos lucros, foi positivo (HAMBRICK et al., 1996). Do mesmo modo, as seguradoras de vida conduzidas por equipes de gestão mais diversificadas superam as seguradoras de vida com equipes de gestão mais homogêneas (PULEO et al., 1998).

Dos 42 artigos, apenas os de Gallén (2006) analisou aspectos distintos dos outros, sendo que o primeiro trabalhou com a sensação, intuição, pensamento e sentimento, e o segundo com propensão ao risco, atitudes inovadoras e habilidades de comunicação. O estilo cognitivo dos gestores e, particularmente, sua forma de captar informações (sensação ou intuição) têm efeito nas estratégias. Gerentes intuitivos tendem a ver o prospector ou a estratégia do analisador como a alternativa futura mais viável para uma empresa. $O$ analisador ou a estratégia de defensor é preferido pelos gerentes de detecção (GALLÉN, 2006).

Ao estudar 46 membros de 8 EGT de 5 empresas, Kauer et al. (2007) encontraram que a diversidade de experiência afeta a definição da agenda e a geração de alternativas, mas - inesperadamente - não parece afetar a velocidade de tomada de decisão. Fatores de personalidade como flexibilidade, motivação de realização, habilidades de rede e orientação de ação parecem ter um impacto mais claro na velocidade de decisão da equipe.

\section{Sugestões para futuras pesquisas}

Outras pesquisas também poderiam visar obter uma compreensão de características não demográficas chave de gerentes individuais que se relacionam com a tomada de decisão estratégica. Medição direta da personalidade subjacente e variáveis cognitivas, por exemplo, iria adicionar insights para a questão da natureza das interações de equipe e os resultados da decisão (BANTEL, 1993).

Verificou-se que pesquisas envolvendo a Teoria dos Escalões Superiores carecem de em focar no fator ambiental como variável explicativa (GOLL et al., 2008; PING, 2008; DÍAZ-FERNÁNDEZ et al., 2014; PING et al., 2016). Tal análise pode contribuir para uma compreensão mais rica da tomada de decisões estratégicas nas organizações além de, pelo menos em parte, explicar alguns dos achados fracos ou conflitantes de pesquisas anteriores (GOLL et al., 2008). De acordo com Ping (2008), o ambiente externo das empresas, as características da indústria e a estrutura de propriedade podem afetar as relações e, por tal motivo, devem ser levados em consideração em futuras pesquisas.

Poucos estudos foram realizados exploram a diferença cultural dos gestores. Os resultados da pesquisa feita em com gestores das subsidiárias listadas na America's Corporate Families and International Affiliates mostram que a heterogeneidade cultural afeta tanto os processos e o desempenho da EGT quanto o desempenho da organização de que o EGT é responsável - a subsidiária no exterior (ELRON, 1997). A pesquisa futura deve explorar relação entre as diferentes culturas (ELRON, 1997; DÍAZ-FERNÁNDEZ et al., 2014; PING et al., 2016).

Há a necessidade de considerar determinantes adicionais de mudanças nos padrões de atenção gerencial, além das características da equipe de gestão. Uma segunda oportunidade de pesquisa é examinar não apenas o grau, mas também a velocidade, da mudança atencional. Finalmente, não podemos deixar de nos encorajar pelo potencial da análise automática de texto para medir a atenção gerencial (CHO et al., 2006). 
Identificam-se oportunidades de explorar características como gênero e etnia. A heterogeneidade do grupo (gênero ou etnia), contrariamente à previsão, foi caracterizada por ter impacto negativo na extensão da adoção de tecnologia da informação (CHUANG et al., 2007; CHUANG et al., 2009). Na pesquisa de Awa et al. (2011) feita com 432 gestores de Pequenas e Médias Empresas da Nigéria, a sensibilidade ao gênero de EGT membros das Pequenas e Médias Empresas foram encontrados para ter poder significativamente forte de prever a extensão da adoção de tecnologia da informação.

Também se encontrou a oportunidade de estender os estudos da TES para outras áreas, tais como finanças e gestão da operação (YUAN et al., 2014). Inclui-se a análise de sistemas de contabilidade, bem como o efeito combinado dos escalões superiores e da contabilidade gerencial e sistemas de controle do desempenho organizacional (HIEBL, 2014).

Estudos futuros podem reunir as variáveis cognitivas dos membros da equipe, como relação de poder e confiança e influência entre membros, por meio de dados de pesquisa para adquirir uma compreensão mais profunda dos processos cognitivos em resultados estratégicos (ELRON, 1997; ATHANASSIOU et al., 1999; LI, 2016). Além de medir as variáveis de composição da EGT, sugere-se que próximas pesquisas também utilizem instrumentos para avaliar os processos psicológicos subjacentes (ACAR, 2016).

Desde a década de 1990, pesquisadores perceberam que não era apropriado apenas usar o método demográfico, que tinha muitos fatores limitados (WEIHUI et al., 2008). A abordagem demográfica tem sido criticada com o argumento de que a abordagem não tem acesso à 'caixa preta' - os processos que ligam os fatores demográficos e os resultados organizacionais (LAWRENCE, 1997). Adicionalmente, Pettigrew (1992) argumentou que o conhecimento acerca dos processos pelos quais as principais equipes realizam suas tarefas é bastante incompleto.

Mas há dois principais motivos inter-relacionados pelo qual os investigadores não estudam os processos psicológicos e sociais que transformar características dos executivos em ação estratégica. Em primeiro lugar, há relativamente poucos pesquisadores que têm interesse e facilidade com ambos os microprocessos e fenômenos macroorganizacionais. Em segundo lugar, esta pesquisa da caixa preta não foi feita porque é extremamente difícil (HAMBRICK, 2007). Por desfecho, considera-se complicado encontrar executivos para medir as variáveis psicológicas ou dinâmicas da equipe, que podem ser as características de processo mais importantes que ligam os atributos da equipe de gestão superior aos resultados organizacionais (WEIHUI et al., 2008).

\section{CONCLUSÕES}

Este artigo traçou a trajetória científica das publicações da Teoria dos Escalões Superiores (TES), desde seus antecedentes até suas publicações empíricas de 2017. Isso permitiu identificar a origem da Teoria e as perspectivas subsequentes. Notou-se que a Teoria é constituída com origem na Teoria Comportamental, sobretudo pela característica da racionalidade limitada que os gestores possuem para lidar com os problemas de negócio. Sobre o aspecto da escolha estratégica, está a relação das escolhas dos gestores com o desempenho da empresa, uma vez que as escolhas gerenciais desempenham um papel importante nas ações 
e resultados organizacionais.

Uma premissa diferencial da Teoria dos Escalões Superiores é o foco primário na coalizão dominante, isto é, equipe de alta gerência, ao invés de estritamente no executivo-chefe. Dentro dessa premissa Song (1982) e Pfeffer (1983) reconhecerem que as variáveis demográficas dos gestores tinham potencial como variáveis causais no estudo dos resultados organizacionais. Adicionalmente, a Visão Baseada em Recursos (VBR) contribuiu com o entendimento de que a equipe de direção figura como um recurso estratégico da empresa.

Hambrick et al. (1984) compilaram essas diversas ideias na construção da perspectiva de escalões superiores. Para eles, os resultados organizacionais são reflexos das escolhas estratégicas de seus principais executivos e essas escolhas são filtradas pelas variáveis demográficas. Na trajetória precedente das publicações, constatou-se o acréscimo de estudos realizados envolvendo a TES de maneira progressiva nas últimas décadas. De certo modo, indica-se o do interesse dos pesquisadores em verificar a influência das características dos gestores em diversos aspectos.

$\mathrm{Na}$ análise dos artigos empíricos foram encontradas dez características usuais entre os trabalhos referentes a TES, com destaque para a educação e a heterogeneidade/homogeneidade da Equipe de Gestão de Topo. Desde a década de 1990 os pesquisadores identificaram que não era apropriado apenas usar o método demográfico, que tinha muitos fatores limitados (WEIHUI et al., 2008). Apesar de abordagem demográfica ser criticada com o argumento de que a abordagem não tem acesso aos processos que ligam os fatores demográficos aos resultados organizacionais, a realização deste tipo de pesquisa é bastante difícil.

Sugerem-se pesquisas que incluam a variável ambiental na aplicação da TES, explorem as diferenças culturais, gênero e etnia entre gestores e/ou EGT, além de estender os estudos da TES para outras áreas, tais como finanças e gestão da operação. Por fim, ainda se observa a necessidade de análise de processos psicológicos e sociais das EGT.

\section{REFERÊNCIAS}

ACAR, F. P.. The effects of top management team composition on SME export performance: an upper echelons perspective. Central European Journal of Operations Research, v.24, p.833-852, 2016. DOI: http://doi.org/10.1007/s10100-015-0408-5

ALDERSON, S.. Reframing management competence: focusing on the Top Management Team. Personnel Review, v.22, n.6, p.53-62, 1993. DOI:

http://doi.org/10.1108/EUM0000000000815

ANDREWS, K. R.. The concept of corporate strategy. Homewood: Dow-Jones-Irwin, 1971.

ATHANASSIOU, N.; NIGH, D.. The impact of US company internationalization on top management team advice networks: A tacit knowledge perspective. Strategic Management Journal, v.20, p.83-92, 1999. Dol: http://doi.org/10.1002/(SICl)10970266(199901)20:1<83::AID-SMJ10>3.0.CO;2-Y

AWA, H. O.; EZE, S. C.; URIETO, J. E.; INYANG, B. J.. Upper echelon theory (UET). Journal of Systems and Information Technology, v.13, n.2, p.144-162, 2011. DOI: http://doi.org/10.1108/13287261111135981

BANTEL, K. A.. Strategic clarity in banking: Role of top management-team demography. Psychological Reports, v.73, p.1187-1201, 1993. DOI: http://doi.org/10.2466/pr0.1993.73.3f.1187

BANY-ARIFFIN, A. N.; MCGOWAN, C. B.; JÚNIOR TUNDE, M. B.; SHAHNAZ, A. S.. Top management characteristics and firm's international diversification activities: evidence from a Developing Nation. Pertanika Journal of Social Sciences \& Humanities, v.22, p.39-54, 2014.

BARNEY, .J. Firm resources and sustained competitive advantage. Journal of Management, v.17, p.99-120, 1991. DOI: http://doi.org/10.1177/014920639101700108

CAMELO, C.; FERNÁNDEZ-ALLES, M.; HERNÁNDEZ, A. B.. Strategic consensus, top management teams, and innovation performance. International Journal of 
Manpower, v.31 n.6, p.678-695, 2010. DOI: https://doi.org/10.1108/01437721011073373

CARPENTER, M. A.; FREDRICKSON, J. W.. Top management teams, global strategic posture and the moderating role of uncertainty. Academy of Management Journal, v.23, p.275284, 2001. DOI: http://doi.org/10.2307/3069368

CARPENTER, M. A.; GELETKANYCZ, M. A.; SANDERS, G. W. Upper echelons research revisited: antecedents, elements, and consequences of top management team composition. Journal of Management, v.30, n.6, p.749-778, 2004. DOI: http://doi.org/10.1016/j.jm.2004.06.001

CARPENTER, M. A.; REILLY, G. P.. Constructs and Construct Measurement in Upper Echelons Research. Research Methodology in Strategy and Management, v.3, p.17-35, 2006. DOI: http://doi.org/10.1016/S1479-8387(06)03003-7

CASTANIAS, R. P.; HELFAT, C. E.. The managerial rents model: Theory and empirical analysis. Journal of Management, v.27, n.1, p.661-678, 2001. DOI: http://doi.org/10.1016/S0149-2063(01)00117-9

CHANDLER, A.. Strategy and Structure: Chapters in the History of the Industrial Enterprise. Cambridge: MIT Press, 1962.

CHILD, J.. Organizational structure, environment and performance: the role of strategic choice. Sociology, v.6, n.1, p.2-22, 1972. DOI: http://doi.org/10.1177/003803857200600101

CHO, T. S.; HAMBRICK, D. C.. Attention as the mediator between top management team characteristics and strategic change: the case of airline deregulation. Organization Science, v.17, n.4, p.453-469, 2006. DOI: https://doi.org/10.1287/orsc.1060.0192

CHUANG, T. T.; NAKATANI, K.; ZHOU, D.. An exploratory study of the extent of information technology adoption in SMEs: an application of upper echelon theory. Journal of Enterprise Information Management, v.22, n.1-2, p.183196, 2009. DOI: http://doi.org/10.1108/17410390910932821

CHUANG, T. T.; NAKATANI, K.; ZHOU, D.. The impact of managerial characteristics of top management team on the extent of information technology adoption: An exploratory study with the upper echelon theory. In: AMERICAS CONFERENCE ON INFORMATION SYSTEMS, 13. Anais. Praga, 2007.

CLARK, E. D.; SOULSBY, A.. Understanding Top Management and organizational change through demographic and processual analysis. Journal of Management Studies, v.44, n.6, p.932-954, 2007. DOI: http://doi.org/10.1111/i.14676486.2007.00692.x

CYERT, R. M.; MARCH, J. G.. A behavioral theory of the firm. New Jersey: Prentice-Hall, 1963.

DEARBORN, D. C.; SIMON, H. A.. Selective perceptions: A note on the departmental identification of executives. Sociometry, v.21, p.140-144, 1958. DOI: http://doi.org/10.2307/2785898
DENG, Y. Y.. Effects of top management teams on strategic adaptation in new ventures. In: INTERNATIONAL WORKSHOP ON EDUCATION TECHNOLOGY AND TRAINING AND INTERNATIONAL WORKSHOP ON GEOSCIENCE AND REMOTE SENSING, 2. Anais. Shangai, 2008.

DÍAZ-FERNÁNDEZ, M. C.; GONZÁLEZ-RODRÍGUEZ, M. R.; PAWLAK, M.. Top management demographic characteristics and company performance. Industrial Management and Data Systems, v.114, n.3, p.365-386, 2014. DOI: http://doi.org/10.1108/IMDS-04-2013-0210

DUTRA, A.; RIPOLL-FELIU, V.M.; FILLOL, A. G.; ENSSLIN, S. R.; ENSSLIN, L.. The construction of knowledge from the scientific literature about the theme seaport performance evaluation. International Journal of Productivity and Performance Management, v.64, n.2, p.243-269, 2014. DOI: http://10.1108/IJPPM-01-2014-0015

ELRON, E.. Top Management Teams within Multinational Companies: Effects of Cultural Heterogeneity. Journal of International Business Studies, v.8, n.4, p.393-412, 1997. DOI: http://doi.org/10.1016/S1048-9843(97)90021-7

ENSSLIN, S. R.; ENSSLIN, L.; IMLAU, J. M.; CHAVES, L. C.. Processo de mapeamento das publicações científicas de um tema: portfólio bibliográfico e análise bibliométrica sobre avaliação de desempenho de cooperativas de produção agropecuária. Revista de Economia e Sociologia Rural, v.52, p.587-608, 2014. DOI: http://doi.org/10.1590/S010320032014000300010

FINKELSTEIN, S.; HAMBRICK, D. C.. Strategic leadership: top executives and their effects on organizations. Saint Paul: West Publishing, 1996.

FINKELSTEIN, S.; HAMBRICK, D. C.. Top management team tenure and organizational outcomes: the moderating role of managerial discretion. Administrative Science Quarterly, v.35, n.3, p.484-503, 1990. DOI: http://doi.org/10.2307/2393314

GALLÉN, T.. Managers and strategic decisions: does the cognitive style matter?. Journal of Management Development, v.25, n.2, p.118-133, 2006. DOI: http://doi.org/10.1108/02621710610645117

GOLL; I.; JOHNSON; N. B.; RASHEED, A.. Top management team demographic characteristics, business strategy, and firm performance in the US airline industry - The role of managerial discretion. Management Decision, v.46, n.2, p.201-222, 2008. DOI: http://doi.org/10.1108/00251740810854122

GOLL, I.; RASHEED, A.. The relationships between top management demographic characteristics, rational decision making, environmental munificence, and firm performance. Organization Studies, v.26, p.999-1023, 2005. DOI: http://doi.org/10.1177/0170840605053538

GUO, R. LIU, B.; YANG, Z.-L.. Research on decision-making effectiveness factors of top management team. In: INTERNATIONAL CONFERENCE ON MANAGEMENT SCIENCE \& ENGINEERING, 18. Anais. Roma, 2011.

HAMBRICK, D. C.. Top management teams: Key to strategic success. California Management Review, v.30, p.88-108, 
1987. DOI: http://doi.org/10.2307/41165268

HAMBRICK, D. C.. Upper echelons theory: an update. The Academy of Management Review, v.32, n.2, p.334-343, 2007. DOI: http://doi.org/10.2307/20159303

HAMBRICK, D. C.; CHO, T. S.; CHEN, M-J.. The influence of top management team heterogeneity on firms' competitive moves. Administrative Science Quarterly, v.41, n.4, p.659684, 1996. DOI: http://doi.org/10.2307/2393871

HAMBRICK, D. C.; MASON, P. A.. Upper echelons: The organization as a reflection of its top managers. Academy of Management Review, v.9, p.193-206, 1984. DOI: http://doi.org/10.2307/258434

HANNAN, M.; FREEMAN, J.. The population ecology of organizations. American Journal of Sociology, v.82, p.929964, 1977. DOI: http://doi.org/10.1086/226424

HAO, Y.; YUE, C. T.. The influence of strategic orientation on innovation performance: A research framework from TMT perspective. In: INTERNATIONAL SYMPOSIUM ON MANAGEMENT OF TECHNOLOGY. Anais. Hangzhou, 2012.

HERRMANN, P.; DATTA, D. K.. Relationships between top management team characteristics and international diversification: an empirical investigation. British Journal of Management, v.16, p.69-78, 2005. DOI: http://doi.org/10.1111/i.1467-8551.2005.00429.x

HIEBL, M. R. W.. Upper echelons theory in management accounting and control research. Journal of Management Control, v.24, n.3, p.223-240, 2014. DOI: http://doi.org/10.1007/s00187-013-0183-1

JEHN, K.; NORTHCRAFT, G. B.; NEALE, M. A.. Why differences make a difference: a field study of diversity, conflict and performance in workgroups. Administrative Science Quarterly, v.44, n.4, p.741-763, 1999. DOI: http://doi.org/10.2307/2667054

KAUER, D.; WALDECK, T. C. P.; SCHÄFFER, U.. Effects of top management team characteristics on strategic decision making. Management Decision, v.45, n.6, p.942-967, 2007.

KRISHNAN, H. A.; PARK, D.. The influence of top management team leadership on corporate refocusing: $A$ theoretical framework. Journal of Leadership and Organizational Studies, v.5, n.2, p.50-61, 1998. DOI: http://doi.org/10.1177/107179199900500206

LAWRENCE, B. S.. The black box of organizational demography. Organization Science, v.8, p.1-22, 1997. DOI: http://doi.org/10.1287/orsc.8.1.1

LI, P. Y.. The impact of the top management teams' knowledge and experience on strategic decisions and performance. Journal of Management and Organization, v.22, p.1-20, 2016a. DOI: http://doi.org/10.1017/jmo.2016.24

LI, W.; ZHANG, S.; ZHANG, J.. Top management team heterogeneity, diversification, and corporate performance: A panel smooth transition regression model. Romanian Journal of Economic Forecasting, v.18, n.1, p.129-142, 2015.
MARCH, J. G.; SIMON, H. A.. Organizations. New York: Wiley, 1958.

MICHEL, J. G.; HAMBRICK, D. C.. Diversification posture and top management team characteristics. Academy of Management Journal, v.35, n.1, p.9-37, 1992. DOI: http://doi.org/10.2307/256471

NARANJO-GIL, D.; HARTMANN, F.; MAAS, V. S.. Top management team heterogeneity, strategic change and operational performance. British Journal of Management, v.19, p.222-234, 2008. DOI: http://doi.org/10.1111/j.14678551.2007.00545.x

PENROSE, E. T.. The theory of the growth of the firm. New York: Wiley, 1959.

PETTIGREW, A. M.. On studying managerial elites. Strategic Management Journal, v.13, p.163-182, 1992. DOI: http://doi.org/10.1002/smj.4250130911

PFEFFER, J.. Organizational demography. In: CUMMINGS, L. L.; STAW, B. M.. Research in organizational behavior. Greenwich: JAI Press, 1983. p.299-357.

PING, Z.. Research on Top Management Team heterogeneity and strategic changes in the transition economy: an empirical research on Chinese Listed. In: INTERNATIONAL CONFERENCE ON WIRELESS COMMUNICATIONS NETWORKING AND MOBILE COMPUTING, 4. Anais. Dalian, 2008.

PING, G.; FENG, W.; YANYAN, Z.. A Review of Enterprise Top Management Team and Organizational Innovation. In: INTERNATIONAL CONFERENCE ON INNOVATION AND MANAGEMENT, 13. Anais. Wuhan, 2016.

PULEO, V. A.; MARLIN, D.. Top management team characteristics and life insurer performance: do top managers make a difference?. Risk Management and Insurance Review, v.2, n.1, p.24-36, 1998. DOI: http://doi.org/10.1111/i.1540-6296.1998.tb00080.x

SERRA, B. P. C.; TOMEI, P. A.; SERRA, F. A. R.. A pesquisa em tomada de decisão estratégica no alto escalão: evolução e base intelectual do tema. Revista de Ciências da Administração, v.16, n.40, p.11-28, 2014. DOI: http://doi.org/10.5007/2175-8077.2014v16n40p11

SIMON, H. A.. A behavioral model of rational choice. The quarterly Journal of Economics, v.69, n.1, p.99-118, 1955. DOI: http://doi.org/10.2307/1884852

SONG, J.. Diversification strategies and the experience of top executives of large firms. Strategic Management Journal, v.3, p.377-380, 1982. DOI: http://doi.org/10.1002/smi.4250030411

THOMAS, A.; LITSCHERT, R.; RAMASWAMY, K.. The performance impact of strategy-manager co-alignment: an empirical examination. Strategic Management Journal, v.12, n.7, p.509-522, 1991. DOI: http://doi.org/10.1002/smj.4250120704

WALLY, S.; BECERRA, M.. Top management team characteristics and strategic changes in international diversification. Group \& Organization Management, v.26, 
n.2, p.165-188, 2001. DOI:

http://doi.org/10.1177/1059601101262003

WALSH, J. P.. Selectivity and selective perception: An investigation of managers' belief structures and information processing. Academy of Management Journal, v.31, p.873896, 1988. DOI: http://doi.org/10.2307/256343

WEIHUI, D.; YUE, Y.. Multi-Agent Model and Decision Support System for Top Management Team. In: INTERNATIONAL CONFERENCE ON WIRELESS COMMUNICATIONS, NETWORKING AND MOBILE COMPUTING, 4. Anais. Nova lorque, 2008.

WERNERFELT, B.. A resource-based view of the firm. Strategic Management Journal, v.5, p.171-180, 1984. DOI: http://doi.org/10.1002/smj.4250050207
WIERSEMA, M. P.; BANTEL, K. A.. Top management team demography and corporate strategic change. Academy of Management Journal, v.35, p.91-121, 1992. DOI: http://doi.org/10.2307/256474

YUAN, X.; GUO, Z.; FANG, E.. An examination of how and when the top management team matters for firm innovativeness: The effects of TMT functional backgrounds, Innovation: Management. Policy \& Practice, v.16, n.3, p.323342, 2014. DOI:

http://doi.org/10.1080/14479338.2014.11081991

YU-HONG, A.; BING, L.; YUAN, L.. Mechanism of action of TMT managerial discretion to strategic decision. In: INTERNATIONAL CONFERENCE ON MANAGEMENT SCIENCE AND ENGINEERING, 20. Anais. Harbin, 2013.

A CBPC - Companhia Brasileira de Produção Científica (CNPJ: 11.221.422/0001-03) detém os direitos materiais desta publicação. Os direitos referem-se à publicação do trabalho em qualquer parte do mundo, incluindo os direitos às renovações, expansões e disseminações da contribuição, bem como outros direitos subsidiários. Todos os trabalhos publicados eletronicamente poderão posteriormente ser publicados em coletâneas impressas sob coordenação da Sustenere Publishing, da Companhia Brasileira de Produção Científica e seus parceiros autorizados. Os (as) autores (as) preservam os direitos autorais, mas não têm permissão para a publicação da contribuição em outro meio, impresso ou digital, em português ou em tradução. 